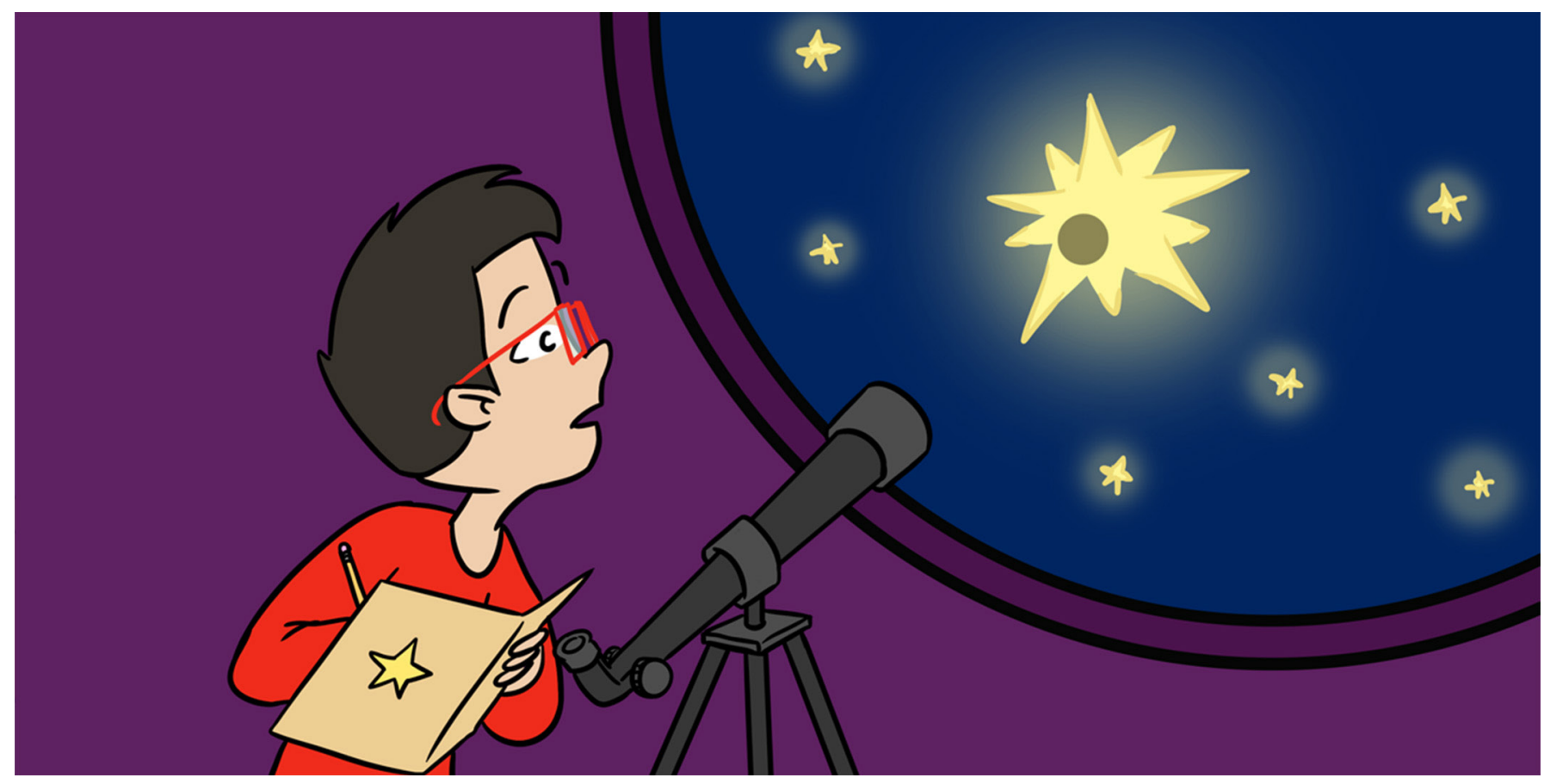

\title{
HOW TO DISCOVER A PLANET ORBITING A DISTANT STAR
}

\section{Brett M. Morris*}

Department of Astronomy, University of Washington, Seattle, WA, United States

YOUNG REVIEWER:

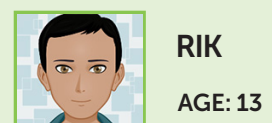

Recently, thousands of planets have been discovered orbiting distant stars. We call these far-away planets exoplanets. Most exoplanets have been found by looking for tiny changes in the brightness of stars. When exoplanets pass in between their star and the Earth, they block out a bit of starlight, causing a small dip in the star's brightness. Transits of exoplanets have revealed that the most common planets orbiting Sun-like stars are a bit bigger than the Earth and smaller than Neptune-unlike any planet in our Solar System.

\section{WHY IS IT SO HARD TO FIND PLANETS ORBITING DISTANT STARS?}

We have known about the planets orbiting the Sun in our own solar system for thousands of years, but it took until 1995 to find a planet orbiting a distant Sun-like star. Finding planets orbiting distant starts is difficult because planets are much dimmer than the stars they orbit, 


\section{LIGHT YEAR}

The distance that light travels in 1 year.

\section{TRANSIT}

When a planet appears to block out its star's light.

\section{Figure 1}

This figure shows brightness measurements over time for the star HD 209458. You can see that the brightness dipped below its normal brightness for a while before returning to its normal brightness -the dip in brightness is the transit event which occurs because the Jupiter-sized exoplanet orbiting HD 209458 is blocking out some of the starlight [1]. and both the stars and planets are so far away. It is a bit like trying to see a mosquito at night, flying next to a car's headlight, from 100 miles away! So how in the world do we find other worlds?

\section{ONE LITTLE ECLIPSE MAKES ONE BIG BREAKTHROUGH}

A breakthrough came in the year 2000, when astronomers pointed their telescope at a star called HD 209458. HD 209458 is slightly bigger and hotter than the Sun, and is located about 150 light years away, in the constellation Pegasus. The astronomers were measuring the brightness of the star very carefully-with enough precision to detect changes in the star's brightness as small as $1 \%$. They measured the star's brightness on two different nights and found that the star got dimmer by about $1.5 \%$ for $3 \mathrm{~h}$, before returning to its normal brightness, on two separate nights [1].

That tiny change in brightness, shown in Figure 1, was a huge discovery, because it was caused by a planet that passed between the Earth and HD 209458. As the planet blocked out starlight from HD 209458, the astronomers on Earth measured slightly less starlight than normal. The amount of missing starlight tells astronomers how big the planet is. If the planet is very large, it blocks out lots of starlight, and if the planet is smaller, like Earth, it blocks only a tiny fraction of the star's light. When a planet passes in front of a star and blocks out starlight, we call that event a transit.

The amount of light that went missing from HD 209458 (1.5\%) must have been caused by a planet bigger than Jupiter-the biggest planet in our solar system. They called the exoplanet HD 209458 b. The little $b$ represents that it was the first planet discovered in the system (if later planets are discovered around the same star, they will be given the letters $c$, then d, and so on). Though its size is similar to that of Jupiter, HD

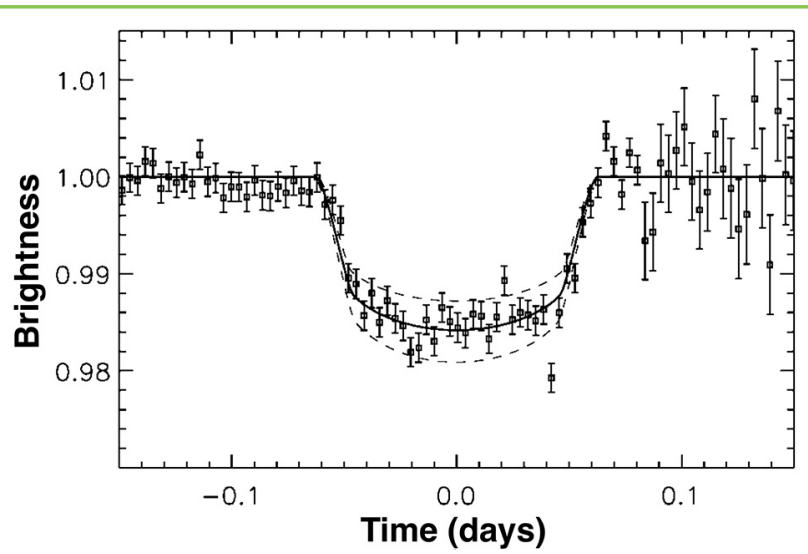

Figure 1 
KEPLER SPACE

\section{TELESCOPE}

A space telescope in orbit around the Sun, which hunts for exoplanets using the transit method.
209458 b is very different from Jupiter. Jupiter orbits the Sun five times farther than the distance from the Earth to the Sun, while HD 209458 b orbits its star 21 times closer than Earth. Since HD 209458 b is so close to its star, it is extremely hot-about $1,200^{\circ} \mathrm{C}$ ! Like Jupiter, this planet is a "gas giant," meaning it has no solid surface. Without a surface and at such a high temperature, HD 209458 b is a bad place to look for life.

\section{FINDING THOUSANDS OF PLANETS}

Now we know how to discover one planet-we measure the brightness of a star very carefully for a while, and if it dips in brightness for a few hours during a transit, we may have discovered a planet! But since the year 2000, thousands of planets have been discovered. How did astronomers find so many?

Most of the planets discovered so far have been found by the Kepler space telescope, which stared at 150,000 stars for 4 years, taking precise brightness measurements every half hour. More than 2,000 stars showed transits in the brightness measurements, revealing the presence of a whole zoo of planets that range in size from as small as Mercury to larger than Jupiter, and everything in between [2].

\section{THE MEDIUM-SIZED PLANET SURPRISE}

One of the biggest surprises that the Keplerspace telescope discovered was that the most common size for a planet is in between the size of Earth and Neptune. Earth is the largest rocky planet in the solar system, and Neptune is the smallest gas planet in the solar system. There are no planets in our solar system between the size of Earth and Neptune, yet most of the planets found by Keplerfall in this size range. Astronomers today are working hard to figure out what these medium-sized worlds are like [3]. Are they small gas planets like mini-Neptunes, or big, rocky planets like super-Earths?

\section{NOT TOO HOT, AND NOT TOO COLD-JUST RIGHT!}

You might be wondering, "Could we live on any of those planets?" Astronomers are trying to answer that question. More specifically, they want to know whether or not each planet could have liquid water on its surface. Everywhere liquid water is found on the Earth, life can also be found, so we will begin our search for life among the exoplanets by searching for planets that could have liquid water on their surfaces. Some planets, like HD 209458 b, are too hot to have liquid water, while 


\section{HABITABLE ZONE}

Region around a star where it is not too hot and not too coldwhere water would be liquid on the surface of a rocky planet. others are too cold. We are looking for planets that sit in the "Goldilocks zone," right in the middle, which we call the "habitable zone."

Two planets that orbit in the habitable zone around their host star are Kepler-62 e and $f[4,5]$. These planets orbit a star $30 \%$ less massive than the Sun, located 1,200 light years away from us. Both planets are a bit larger than the Earth. Astronomers hope to use the soon-to-be-launched James Webb space telescope to measure the masses of these nearly Earth-sized worlds, to figure out if they are rocky or gaseous, and therefore whether or not they have surfaces (and possibly water) and would be good hosts to life as we know it.

\section{NEW PLANET HUNTERS}

There are a variety of exciting new missions being planned by NASA and ESA that will discover or study transiting exoplanets, including missions called TESS, PLATO, and CHEOPS. As we discover more and more planets, we will surely need more astronomers like you to help us figure out which ones are the most promising hosts for life.

\section{REFERENCES}

1. Charbonneau, D., Brown, T. M., Latham, D. W., and Mayor, M. 2000. Detection of planetary transits across a sun-like star. Astrophys. J. 529:L45. doi: $10.1086 / 312457$

2. Akeson, R. L., Chen, X., Ciardi, D., Good, J., Harbut, M., Jackson, E., et al. 2013. The NASA exoplanet archive: data and tools for exoplanet research. Publ. Astron. Soc. Pac. 125:989. doi: 10.1086/672273

3. Rogers, L. A. 2015. Most 1.6 earth-radius planets are not rocky. Astrophys. J. 801:41. doi: 10.1088/0004-637X/801/1/41

4. Borucki, W. J., Agol, E., Fressin, F., Kaltenegger, L., Rowe, J., Isaacson, H., et al. 2013. Kepler-62: a five-planet system with planets of 1.4 and 1.6 earth radii in the habitable zone. Science 340:587-90. doi: 10.1126/science.1234702

5. Shields, A. L., Barnes, R., Agol, E., Charnay, B., Bitz, C., and Meadows, V. S. 2016. The effect of orbital configuration on the possible climates and habitability of Kepler-62f. Astrobiology 16:443-64. doi: 10.1089/ast.2015.1353

SUBMITTED: 11 October 2018; ACCEPTED: 06 December 2018; PUBLISHED ONLINE: 08 January 2019.

EDITED BY: Majken Brahe Ellegaard Christensen, Astronomicca, Denmark 
CITATION: Morris BM (2019) How to discover a planet orbiting a distant star. Front. Young Minds 6:74. doi: 10.3389/frym.2018.00074

CONFLICT OF INTEREST STATEMENT: The author declares that the research was conducted in the absence of any commercial or financial relationships that could be construed as a potential conflict of interest.

COPYRIGHT @ 2019 Morris. This is an open-access article distributed under the terms of the Creative Commons Attribution License (CC BY). The use, distribution or reproduction in other forums is permitted, provided the original author(s) and the copyright owner(s) are credited and that the original publication in this journal is cited, in accordance with accepted academic practice. No use, distribution or reproduction is permitted which does not comply with these terms.

\section{YOUNG REVIEWER}

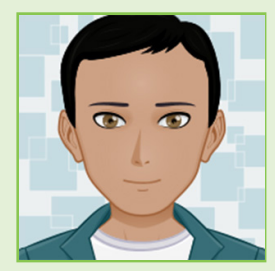

\section{RIK, AGE: 13}

My name is Rik and I am $(5 \times 3)-2$ years old. I guess that tells you I am a math nerd. Learning the guitar, playing soccer, and acting are also some of my favorite things to do.

\section{AUTHOR}

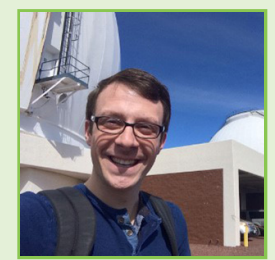

\section{BRETT M. MORRIS}

I am a Ph.D. candidate at the University of Washington studying Astronomy and Astrobiology. Before that, I studied Astronomy and Physics at the University of Maryland and grew up on Long Island in New York. I always wanted to be an Astronomer, and I am almost there! *morrisbrettmagmail.com 